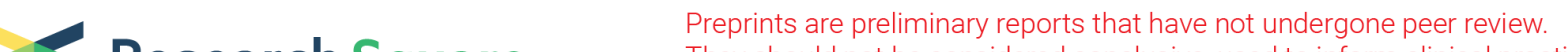 Research Square
or referenced by the media as validated information.
}

\section{High Temperature Superfluorescence in Methyl Ammonium Lead lodide}

\section{Gamze Findik}

NC State University

Melike Biliroglu

NC State University

Dovletgeldi Seyitliyev

NC State University

Juliana Mendes

North Carolina State University

Andrew Barrette

NC State University

Hossein Ardekani

North Carolina State University

Lei Lei

North Carolina State University https://orcid.org/0000-0002-1160-3689

\section{Qi Dong}

North Carolina State University

\section{Franky So}

North Carolina State University https://orcid.org/0000-0002-8310-677X

Kenan Gundogdu ( $\square$ kgundog@ncsu.edu )

NC State University

\section{Research Article}

Keywords: Light-matter Interactions, Collective Many-body Phases, High Energy Electron-hole Pairs, Macroscopic Quantum Coherence

Posted Date: December 9th, 2020

DOl: https://doi.org/10.21203/rs.3.rs-115325/v1

License: (9) (1) This work is licensed under a Creative Commons Attribution 4.0 International License. Read Full License 
Version of Record: A version of this preprint was published at Nature Photonics on June $21 \mathrm{st}, 2021$. See the published version at https://doi.org/10.1038/s41566-021-00830-x. 


\title{
Title: High Temperature Superfluorescence in Methyl Ammonium Lead
}

\section{Iodide}

Authors: Gamze Findik ${ }^{1 \dagger}$, Melike Biliroglu ${ }^{1 \dagger}$, Dovletgeldi Seyitliyev ${ }^{1}$, Juliana Mendes ${ }^{2}$, Andrew Barrette $^{1}$, Hossein Ardekani ${ }^{1}$, Lei Lei ${ }^{2}$, Qi Dong ${ }^{2}$, Franky So $^{2}$, Kenan Gundogdu ${ }^{1 *}$

\author{
Affiliations: \\ ${ }^{1}$ Department of Physics, North Carolina State University, Raleigh, North Carolina 27695, United \\ States \\ ${ }^{2}$ Department of Materials Science and Engineering, North Carolina State University, Raleigh, \\ North Carolina 27695, United States \\ ${ }^{1,2}$ Organic and Carbon Electronics Laboratory (ORaCEL), North Carolina State University, \\ Raleigh, North Carolina 27695, United States \\ *Correspondence to: kgundog@ncsu.edu \\ $\uparrow$ These authors contributed equally to this work.
}

Light-matter interactions can create and manipulate collective many-body phases in solids ${ }^{1-}$ ${ }^{3}$, which are promising for the realization of emerging quantum applications. However, in most cases these collective quantum states are fragile, with a short decoherence and dephasing time, limiting their existence to precision tailored structures under delicate conditions such as cryogenic temperatures and/or high magnetic fields. In this work, we discovered that the archetypal hybrid perovskite, $\mathrm{MAPbI}_{3}$ thin films, exhibit such a collective coherent quantum many-body phase, namely superfluorescence, at $78 \mathrm{~K}$ and above. Pulsed laser excitation first creates a population of high energy electron-hole pairs, which quickly 
relax to lower energy domains and then develop a macroscopic quantum coherence through spontaneous synchronization. The excitation fluence dependence of the spectroscopic features and the population kinetics in such films unambiguously confirm all the well-known characteristics of superfluorescence. These results show that the creation and manipulation of collective coherent states in hybrid perovskites can be used as the basic building blocks for quantum applications $\mathbf{s}^{4,5}$.

Spontaneous synchronization of oscillators is a fascinating process. While it is best visualized as the buildup of a collective phase in initially randomly oscillating metronomes coupled to the same medium, spontaneous synchronization is a universal phenomenon occurring in natural processes such as the initial ordering of the planetary orbits, frequency locking of triode generators, and signal synchronization of fireflies in the wild ${ }^{6}$. This phenomenon prevails not only in macro and micro classical realms, spanning physical and biological systems, but it also leads to the manifestation of exotic collective quantum phenomena ${ }^{3}$. In the quantum domain, systems are described by wave functions in which the "phase" plays a dominant role as it determines the waveform and its relation to other waves, along with their collective behavior under external stimuli. While an incoherent population of quantum objects has a random distribution of phases, spontaneous synchronization leads to symmetry breaking and the observation of exotic collective quantum phenomena including but not limited to, superconductivity, Bose-Einstein condensation, and the collective dynamics of Josephson junctions ${ }^{7}$.

A remarkable example of spontaneous synchronization is the superfluorescence of optically excited dipoles in a small volume. Figure 1 illustrates the process in which the initially excited population of dipoles has a random phase distribution. The vacuum field interactions spontaneously synchronize the phases of these oscillators. As a result, the system experiences a 
phase transition into the Dicke superradiant state ${ }^{8-10}$. In this state, all the excitations interact with the radiation field collectively and coherently. Acting like a giant atom, they emit a high-intensity short burst of photons. This phase transition of an ensemble of incoherent dipoles into a coherent macroscopic quantum state and its collective radiation is called superfluorescence (SF).

The quantum phase transition that leads to SF depends on the dephasing time of the excitations. Macroscopic coherence can only build up if the dephasing is slower than spontaneous synchronization. Therefore, initial observation of SF has been primarily in gas-phase systems ${ }^{11}$. Due to fast electronic dephasing in condensed matter, SF has only been observed at cryogenic temperatures $(\sim 10 \mathrm{~K})$ in a handful of solids. These include excitonic transitions in oxygen-doped $\mathrm{KCl}^{12}$, localized atomic-like states, such as $\mathrm{CuCl}$ nanocrystals embedded into a $\mathrm{NaCl}$ matrix ${ }^{13}$, and excitonic transitions in bulk ZnTe single crystals ${ }^{14}$. Also, electron-hole plasma in InGaAs quantum wells exhibits SF under a high magnetic field ( $>10$ Tesla) ${ }^{15}$. More recently superfluorescence was observed in $\mathrm{CsPbBr}_{3}$ perovskite nanocrystals at $6 \mathrm{~K}^{16}$. In contrast to previous solid-state systems where low cryogenic temperatures and/or high magnetic field are required to demonstrate superfluorescence, we demonstrate that hybrid perovskite $\mathrm{MAPbI}_{3}$ exhibits SF at temperatures achievable by liquid nitrogen, indicating that the versatile hybrid perovskite material system is an ideal platform to study SF and create collective coherent quantum states of matter suitable for quantum applications at elevated non-cryogenic temperatures.

Since the SF process requires spontaneous synchronization and the emission is from a macroscopic coherent state, it exhibits characteristic spectroscopic signatures that unambiguously help distinguish it from other collective radiation processes, such as amplified spontaneous emission (ASE). These spectroscopic signatures are measurable using steady-state photoluminescence (PL), time-resolved emission, and time-resolved absorption spectroscopies. First and foremost, the 
initial population in SF does not have macroscopic coherence, therefore there is a delay time during which spontaneous synchronization takes place, preceding the formation of a macroscopic coherent state ${ }^{17}$. Secondly, this delay time reduces with an increase in the excitation density ${ }^{17}$. Also, since the emission is from a Dicke superradiant state, the lifetime decreases with the density "N" of phase-locked indistinguishable quantum oscillators ${ }^{17}$. Since all the excitations interact coherently with the radiating field, the maximum intensity of the SF pulse scales with $\mathrm{N}^{2}$, leading to a quadratic dependence on the excitation density. Moreover, SF emission exhibits interference and propagation effects, leading to oscillations called Burnham-Chiao ringing in the time evolution ${ }^{18}$. Last but not least, the emission kinetics of SF is similar to the relaxation of an inverted pendulum ${ }^{19}$; as a result, it has a very specific time-dependent functional form based on "sech", unlike the exponential behavior of spontaneous recombination of individual excitations ${ }^{20}$. Here we show that the hybrid perovskite $\mathrm{MAPbI}_{3}$ exhibits all of these characteristics of SF when excited at and above a certain threshold using ultrafast laser pulses (See Supplementary Section B1).

Figure 2a shows the absorption spectra of $\mathrm{MAPbI}_{3}$ at temperatures ranging from $78 \mathrm{~K}$ to room temperature (RT). It is known that $\mathrm{MAPbI}_{3}$ is in a tetragonal phase (TP) at room temperature and it exhibits a transition to an orthorhombic phase $(\mathrm{OP})$ at temperatures below $150 \mathrm{~K}$. The structural phase transition leads to a blue-shift and a relatively sharp excitonic feature at the band edge of the absorption spectra ${ }^{21}$. It is also well-known that this phase transition is incomplete with a small fraction of TP coexists in the OP domain dominant thin film ${ }^{22}$. Figure $2 b$ shows the continuous wave photoluminescence (CW PL) spectra at $78 \mathrm{~K}$. The sample is excited above the bandgap of the OP domains. The CW PL emission has two distinct features; one at $1.54 \mathrm{eV}$ and the other at $1.58 \mathrm{eV}$, both from the TP domains ${ }^{21,23}$ The absence of OP emission indicates the efficient transfer of excitons to the TP domains ${ }^{22}$. Under CW excitation both features increase superlinearly with 
the fluence, i.e. $I \propto F^{1.4 \pm 0.1}$ (See Supplementary Section A1 and A2). This is because the recombination kinetics is a bimolecular process ${ }^{24}$.

Figure 2c shows the PL spectra when the sample is excited with $120 \mathrm{fs}$ pulses at $400 \mathrm{~nm}$, in which

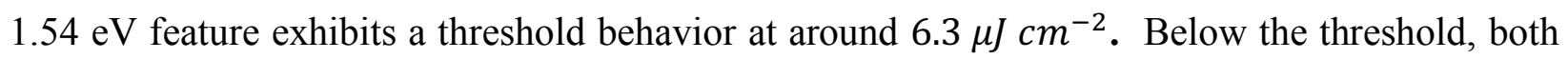
peaks increase linearly (Fig. S7). Beyond the threshold, the $1.54 \mathrm{eV}$ feature exhibits a quadratic increase up to $22.8 \mu \mathrm{J} \mathrm{cm}^{-2}$, while the $1.58 \mathrm{eV}$ feature saturates. The linear increase below the threshold, as opposed to the superlinear increase observed at $\mathrm{CW}$ excitation $(\alpha=1.4 \pm 0.1)$, indicates the existence of excitation density-dependent nonradiative recombination kinetics such as Auger recombination ${ }^{25}$. In contrast, beyond the threshold, the quadratic increase of $1.54 \mathrm{eV}$ feature indicates that a radiative recombination process with a rate much faster than the nonradiative processes takes place. Below we show that this fast recombination process is SF.

We first characterize the time evolution of the sharp feature at $1.54 \mathrm{eV}$ to investigate whether it exhibits the SF dynamics. Figure 3a shows the transient PL kinetics measured at two different above threshold excitation fluences $10.9 \mu \mathrm{J} \mathrm{cm}^{-2}$ and $135.8 \mu \mathrm{J} \mathrm{cm}^{-2}$ (See Fig. S11 and Fig. S12 for all fluences, Supplementary Section C for temperature dependence). The transients in Figs. 3a and S11 have several characteristics that are unique to SF. First, for the range that the integrated PL increases quadratically, the peaks of the PL transients increase superlinearly with fluence $(F)$ with a power-law given by, $I_{\max }=F^{3.9 \pm 0.7}$ (Fig. 3a inset). This observation is consistent with SF because, in a bimolecular process, the rate of exciton formation increases quadratically with the excitation fluence, as a result, the SF peak is expected to increase with the $4^{\text {th }}$ power of the fluence (See Supplementary Section B2 and B3) . Next, we examine the rise and decay characteristics. At low fluence there is no measurable emission for the first 3 picoseconds, then the PL starts to rise slowly. However, as the excitation fluence is increased, the PL signal starts earlier and rises to its 
peak faster. This excitation density-dependent delay in PL rise time is a signature characteristic of SF. The decay also shows faster recombination kinetics as the excitation fluence increases (Fig. 3a), hence the emission takes the shape of a burst with its temporal width (so-called real width) becomes narrower. While conventional exponential functions do not fit these transients, the SF theoretical models based on $\operatorname{sech}^{2}$ functions (Eqs. (S5) and (S8)) fit them (black dashed lines in Fig. 3a) reasonably well ${ }^{20}$ (See Supplementary Section B4). The quantitative values of the delay time " $\tau_{D}$ ", characterizing the time in which spontaneous synchronization takes place, and the real width " $\tau_{R}$ " of the SF burst, obtained from these fits, are displayed in magenta circles in Figs. $3 \mathrm{~b}$ and $3 \mathrm{c}$ respectively. Finally, as fluence increases, the emission exhibits clear recurrences (Fig. S12), known as Burnham-Chiao ringing behavior ${ }^{18}$. All these observations, namely the fluence dependent maximum intensity, delay time, decay, and ringing behavior agree with SF emission.

The delay in emission and its density dependence is a distinctive property of SF and separates it from other collective recombination processes, such as ASE. To further investigate if the delayed emission is due to SF, we first need to show that dynamics related to exciton transfer from orthorhombic to tetragonal domains do not cause the fluence dependent delayed emission. To probe the time evolution of the population in the TP domains, we performed transient absorption spectroscopy. Figure 3 (d-g) shows the pump-probe traces measured at $1.54 \mathrm{eV}$ at $78 \mathrm{~K}$ (See Fig. S14 for all the fluences). The results show that within the first 2 ps of the optical excitation a negative signal occurs and then recovers. This negative feature is a result of bandgap renormalization (BGR) ${ }^{26}$ (See Supplementary Section A3). The population kinetics show that even at the lowest excitation fluence within the first $2 \mathrm{ps}$ after the optical excitation, the population growth in the TP domains is completed and remains steady (See Supplementary Information Fig. S5). However, as the excitation fluence increased beyond the threshold, the pump-probe traces 
show unusual behavior. For instance, at an excitation fluence of $21.5 \mu \mathrm{J} \mathrm{cm}^{-2}$ the traces exhibit similar steady behavior for about 8 ps. But after 8 ps the signal suddenly decays. Interestingly, this waiting period in which the population remains steady depends on the excitation fluence. In Fig. $3 \mathrm{~b}$ the cyan circles are the waiting times measured from the pump-probe experiments and they accurately follow the same trend as extracted $\tau_{D}$ values from the SF model, further confirming that observed kinetics is due to SF. All these observations indicate that the population in the TP domains reaches its maximum value within 2 ps after excitation and the delay in the PL emission is not because excitons did not populate TP domains, but because they need time to establish macroscopic coherence.

According to the SF theory the delay time $\tau_{D}$ scales with $\ln N / N$, and the real width $\tau_{R}$ scales as $1 / N$, where $\mathrm{N}$ is the exciton density ${ }^{17}$ (See Supplementary Section B4 and B5). To do this analysis, one needs to determine the exciton density involved in SF. Exciton density in the TP domains depends both on the excitation fluence and the carrier relaxation dynamics. Since the exciton formation is predominantly bimolecular, the exciton density is proportional to the square of optically excited carrier density (i.e. electron and hole density $n$ ), i.e. $N \propto n^{2}$. Using transient absorption spectroscopy, we determined the excitation fluence dependence of the TP population density right before the SF burst emitted. We found that in the fluence range that the PL behave quadratic, the pump-probe signal at $1.54 \mathrm{eV}$ (TP population) increases linearly with the excitation fluence, i.e. $n \propto F$ (See Supplementary Section B3). Therefore, for this range, exciton density $N$ increases with $F^{2}$. The black solid lines in Figs. $3 \mathrm{~b}$ and $3 \mathrm{c}$ are the $\ln N / N$ and $1 / N$ curves fitting $\tau_{D}$ and $\tau_{\mathrm{R}}$, for the fluence range that population increases linearly.

The population relaxation kinetics measured by the pump-probe experiments are also consistent with the SF kinetics and distinct from the dynamics expected from the ASE process. At very high 
excitation fluences the pump-probe signal rapidly drops (Figs. 3f and 3g). This abrupt drop is similar to that observed in the pump-probe measurements of SF in InGaAs quantum wells studied earlier ${ }^{15}$. As illustrated in Fig. 3h, the electronic levels in the coherent macroscopic system form a Dicke-ladder. In this representation, $\mathrm{N}$ number of excitations coherently exist in the same state. At the top of the Dicke-ladder, all the dipoles are excited and at the bottom, all of them are in the ground state. The decay rate, $\Gamma$, is proportional to $\mathrm{N}$, at the top and the bottom, while at the midlevels it is proportional to $\mathrm{N}^{2}$. This unique fluence dependence of decay rate causes a sudden population drop (Fig. S14), which becomes as fast as $750 \mathrm{fs}$ at $128.9 \mu \mathrm{J} \mathrm{cm}{ }^{-2}$ in Fig. $3 \mathrm{~g}$.

One of the important differences in SF and ASE is that in SF all the coherent population relaxes to the bottom of the Dicke ladder ${ }^{15}$, whereas in ASE the recombination only takes place up until population inversion is lost. As a result, in SF one can observe complete population depletion in contrast to ASE. In our system, the complete depletion of the population in TP domains is hard to observe since there is always a transfer of excess population from the OP domains. As a result, population depletion due to SF and refilling due to transfer from the OP domains compete. A complete population depletion in the TP domains can only be observed when the SF recombination rate exceeds the exciton capture rate by the TP domains significantly. Strikingly, at the highest excitation fluence (Fig. 3g), we observe that the sudden drop (within $750 \mathrm{fs}$ ) in the TP domain population leads to almost complete depletion and then the population rises in a few ps time scale, indicating the cooperative emission is significantly faster than the exciton capture from OP domains, again confirming the observed population kinetics is due to SF.

Having established that the $1.54 \mathrm{eV}$ emission is superfluorescence from TP domains through analysis of the PL and absorption kinetics, we also studied emission directionality to investigate the role of TP domain distribution in SF emission. In extended systems, SF exhibits strong 
directionality determined with the excitation profile ${ }^{27}$. In $\mathrm{MAPbI}_{3} \mathrm{TP}$ domains have random sizes $(100 \mathrm{~nm}-500 \mathrm{~nm})$ and shapes (See Supplementary Information Fig. S1). If multiple TP domains establish a macroscopic coherence, then the directionality should be determined by the excitation profile. In contrast, if SF is originated from individual domains, then the domain sizes and shapes should determine the SF direction. While in the former case SF and ASE should have the same directionality, in the latter case SF direction becomes independent of the excitation profile hence differs from ASE. Our studies show that SF emission from $\mathrm{MAPbI}_{3}$ exhibits a preferentially stronger emission in the forward direction compared to any other direction (Fig. S15). This is true regardless of the size and the shape of the excitation profile (See Supplementary Section D). For instance, in Fig. 4, we show that even when the sample is excited using a stripe-shaped beam with a spot size of $4 \mathrm{~mm} \times 15 \mu \mathrm{m}$, the SF emission at the forward direction is stronger compared to the emission at the edge, which is the optical gain direction. This observation further proves that the observed signal is not ASE, but it is SF radiated from an individual or small clusters of TP domains.

The observation of superfluorescence in solid-state systems has been extremely rare due to stringent requirements to support macroscopic coherence ${ }^{12,14,15}$. Recently, perovskite nanocrystal superlattices based on $\mathrm{CsPbBr}_{3}$ have also been shown to exhibit $\mathrm{SF}$ at $6 \mathrm{~K}^{16}$. Our observation of high-temperature $\mathrm{SF}$ in $\mathrm{MAPbI}_{3}$ thin films suggests that these materials are intrinsically suitable for maintaining quantum coherence. Interesting property in these materials is that optical excitations form strongly bound exciton-polarons. Polaron formation has been known to protect electronic coherence. For instance, in light-harvesting systems, similar polaron formation is found to be a primary reason for sustaining electronic coherence and promoting wave-like energy transfer ${ }^{28}$. In the current example of hybrid perovskites, polaron formation can be the reason for extended 
electronic coherence and observation of macroscopic quantum state. Observation of SF in these versatile materials can make them an ideal platform for quantum applications. We anticipate that these materials can further be used in various microcavities ${ }^{22}$ as building blocks for complex systems where quantum information can be stored, processed, and read out by manipulation and interrogation of giant dipoles ${ }^{4,5,29}$. Finally, from a fundamental point of view, the electronic properties of hybrid perovskites such as the nature of the electronic states, their localization, coupling to phonons modes ${ }^{30}$, and dephasing kinetics, have to be further studied to fully understand the dynamics leading to superfluorescence.

\section{Material and Methods}

\section{Synthesis of $\mathrm{MAPbI}_{3}$ Thin Films:}

Lead iodide (99.999\%), dimethylformamide (anhydrous, 99.8\%), dimethyl sulfoxide (anhydrous, 99.9\%), and toluene (anhydrous, 99.8\%) were purchased from Sigma-Aldrich. Methylammonium iodide was from Dyesol. The perovskite precursor solution was prepared by dissolving $\mathrm{PbI}_{2}$, MAI, and DMSO (the molar ratio is 1:1:1) in DMF with a concentration of $1 \mathrm{M}$. The precursor solution was spin-coated on a glass substrate, during which $100 \mu \mathrm{L}$ of toluene was dropped on the sample at the $8^{\text {th }}$ second. The precursor film was then annealed at $100{ }^{\circ} \mathrm{C}$ for 10 min to complete the crystallization.

\section{Structure Characterization:}

The samples used in this work were $300 \mathrm{~nm}$ thick. The scanning electron microscopy (SEM) image is shown in Fig. S1A. The diagonal line is $5.18 \mu \mathrm{m}$. The average grain size is about $260 \mathrm{~nm}$. Figure S1B shows the atomic force microscopy (AFM) image. The surface roughness (RMS) is $10.6 \mathrm{~nm}$. Figure S1C is the room temperature X-ray diffraction (XRD) data showing the signature (110) peak around 14.8 degrees for the $\mathrm{MAPbI}_{3}$ perovskite-type tetragonal lattice. The other peaks suggest the film is not textured, meaning there is no preferred orientation.

\section{PL experiments:}

A Mightex spectrometer is used to measure the PL. The sample is excited using $400 \mathrm{~nm}$ pulses obtained by frequency doubling of the output of a Ti-Sapphire amplifier with a repetition rate of $1 \mathrm{kHz}$ and the central frequency at $800 \mathrm{~nm}$. For the continuous wave (CW) experiments a $445 \mathrm{~nm}$ InGaN laser is used to excite the sample. 


\section{Kerr-Gate Experiment:}

Time-resolved SF was measured with a homebuilt Kerr-gate set up using a $1 \mathrm{kHz}$ amplified Ti: Sapphire laser (Quantronix Integra-C) with 120 fs pulsed output at $800 \mathrm{~nm}$. As shown in Fig.S2, the laser output beam was split into two paths; one for the optical Kerr gate pulse and the other for the excitation beam, which is converted to $400 \mathrm{~nm}$ through second harmonic generation using a BBO crystal. The collected PL and the gate pulse were focused on $\mathrm{CS}_{2}$ in a $2 \mathrm{~mm}$ thick cuvette, which was used as the Kerr medium. The gated signal is measured with a Hamamatsu photomultiplier tube (H10721-20) attached to a monochromator. The sample is kept in a Janis continuous flow liquid$\mathrm{N}_{2}$ cryostat. The excitation beam size is $1.5 \mathrm{~mm}$.

\section{Time-Resolved Pump-Probe Experiment:}

The pump-probe measurements were performed in a commercial Helios system. The excitation source is an amplified Ti:Sapphire laser (Coherent Libra) system producing $100 \mathrm{fs}$ pulses at $800 \mathrm{~nm}$ at a $1 \mathrm{kHz}$ repetition rate. For the pump path, we frequency double the $800 \mathrm{~nm}$ pulses. Probe pulses are produced by white-light continuum generation in a sapphire crystal.

\section{Time-Correlated Single Photon Counting (TCSPC):}

We used a home-built TCSPC setup with 300 ps time resolution for measuring the longtime PL kinetics. In the TCSPC experiment, the output of a Ti:sapphire amplifier is frequency doubled using a BBO crystal. The PL is collected and guided into a monochromator and detected with a Hamamatsu PMT (H10721-20). 


\section{References:}

1 Torchinsky, D. H., Mahmood, F., Bollinger, A. T., Božović, I. \& Gedik, N. Fluctuating chargedensity waves in a cuprate superconductor. Nature materials 12, 387-391 (2013).

2 Li, T. et al. Femtosecond switching of magnetism via strongly correlated spin-charge quantum excitations. Nature 496, 69-73 (2013).

3 Keeling, J., Marchetti, F., Szymańska, M. \& Littlewood, P. Collective coherence in planar semiconductor microcavities. Semiconductor science and technology 22, R1 (2007).

4 Bergmann, M. \& Gühne, O. Entanglement criteria for Dicke states. Journal of Physics A: Mathematical and Theoretical 46, 385304 (2013).

5 Tóth, G. \& Apellaniz, I. Quantum metrology from a quantum information science perspective. Journal of Physics A: Mathematical and Theoretical 47, 424006 (2014).

6 Whitfield, J. Synchronized swinging. Nature, doi:10.1038/news020218-16 (2002).

7 Eastham, P. R. \& Rosenow, B. Disorder, Synchronization, and Phase-locking in Nonequilibrium Bose-Einstein Condensates. Universal Themes of Bose-Einstein Condensation, 462 (2017).

8 Dicke, R. H. Coherence in spontaneous radiation processes. Physical review 93, 99 (1954).

9 Li, X. et al. Observation of Dicke cooperativity in magnetic interactions. Science 361, 794-797 (2018).

10 Scully, M. O. \& Svidzinsky, A. A. The super of superradiance. Science 325, 1510-1511 (2009).

11 Skribanowitz, N., Herman, I., MacGillivray, J. \& Feld, M. Observation of Dicke superradiance in optically pumped HF gas. Physical Review Letters 30, 309 (1973).

12 Florian, R., Schwan, L. O. \& Schmid, D. Two-color superfluorescence of O2-centers in KCl. Journal of Luminescence 31, 169-171 (1984).

13 Miyajima, K., Kumagai, Y. \& Ishikawa, A. Ultrashort Radiation of Biexcitonic Superfluorescence from High-Density Assembly of Semiconductor Quantum Dots. The Journal of Physical Chemistry C 121, 27751-27757 (2017).

14 Dai, D. \& Monkman, A. Observation of superfluorescence from a quantum ensemble of coherent excitons in a ZnTe crystal: evidence for spontaneous Bose-Einstein condensation of excitons. Physical Review B 84, 115206 (2011).

15 Noe II, G. T. et al. Giant superfluorescent bursts from a semiconductor magneto-plasma. Nature Physics 8, 219-224 (2012).

16 Rainò, G. et al. Superfluorescence from lead halide perovskite quantum dot superlattices. Nature 563, 671-675 (2018).

17 Benedict, M. G. Super-radiance: Multiatomic coherent emission. (CRC Press, 1996).

18 Burnham, D. C. \& Chiao, R. Y. Coherent resonance fluorescence excited by short light pulses. Physical Review 188, 667 (1969).

19 Gross, M. \& Haroche, S. Superradiance: An essay on the theory of collective spontaneous emission. Physics reports 93, 301-396 (1982).

20 Bonifacio, R. \& Lugiato, L. Cooperative radiation processes in two-level systems: Superfluorescence. Physical Review A 11, 1507 (1975).

21 Osherov, A. et al. The impact of phase retention on the structural and optoelectronic properties of metal halide perovskites. Advanced Materials 28, 10757-10763 (2016).

22 Jia, Y., Kerner, R. A., Grede, A. J., Rand, B. P. \& Giebink, N. C. Continuous-wave lasing in an organic-inorganic lead halide perovskite semiconductor. Nature Photonics 11, 784-788 (2017).

23 Chuliá-Jordán, R. et al. Inhibition of light emission from the metastable tetragonal phase at low temperatures in island-like films of lead iodide perovskites. Nanoscale 11, 22378-22386 (2019). 
24 Phuong, L. Q. et al. Free carriers versus excitons in $\mathrm{CH} 3 \mathrm{NH} 3 \mathrm{PbI} 3$ perovskite thin films at low temperatures: charge transfer from the orthorhombic phase to the tetragonal phase. The journal of physical chemistry letters 7, 2316-2321 (2016).

25 Milot, R. L., Eperon, G. E., Snaith, H. J., Johnston, M. B. \& Herz, L. M. Temperature-dependent charge-carrier dynamics in $\mathrm{CH} 3 \mathrm{NH} 3 \mathrm{PbI} 3$ perovskite thin films. Advanced Functional Materials 25, 6218-6227 (2015).

26 Miyata, K. et al. Large polarons in lead halide perovskites. Science Advances 3, e1701217, doi:10.1126/sciadv.1701217 (2017).

27 Jho, Y. et al. Cooperative recombination of a quantized high-density electron-hole plasma in semiconductor quantum wells. Physical review letters 96, 237401 (2006).

28 Ishizaki, A. \& Fleming, G. R. Theoretical examination of quantum coherence in a photosynthetic system at physiological temperature. Proceedings of the National Academy of Sciences 106, 17255-17260 (2009).

29 Wang, Z. et al. Controllable switching between superradiant and subradiant states in a 10-qubit superconducting circuit. Physical Review Letters 124, 013601 (2020).

30 Vardeny, Z. V. Ultrafast dynamics and laser action of organic semiconductors. (CRC Press, 2009).

Acknowledgments: The authors acknowledge the helpful discussions with John Thomas (NC

State University), and David Aspnes (NC State University). The authors also acknowledge

NCSU IMAKS facility and technical support from Dr. Evgeny Danilov for time resolved

absorption experiment. Funding: The authors acknowledge the funding from NSF DMREF

grant \#1729383. Author contributions: GF and MB performed the PL and TRPL measurements

and pump-probe experiments and analyzed the results. DS assisted with the pump-probe and HA

provided help with TR-PL experiments. AB performed steady-state absorption and PL

experiments. LL, QD, FS provided the samples. KG conceived the research problems and

coordinated the studies. KG drafted the manuscript with the help of GF and MB. All authors

helped with editing the manuscript. Competing interests: The authors declare that they have no

competing financial interests. Data availability: All the data included in the manuscript and the

supplementary information are available from the corresponding author upon request. 


\section{Figures}

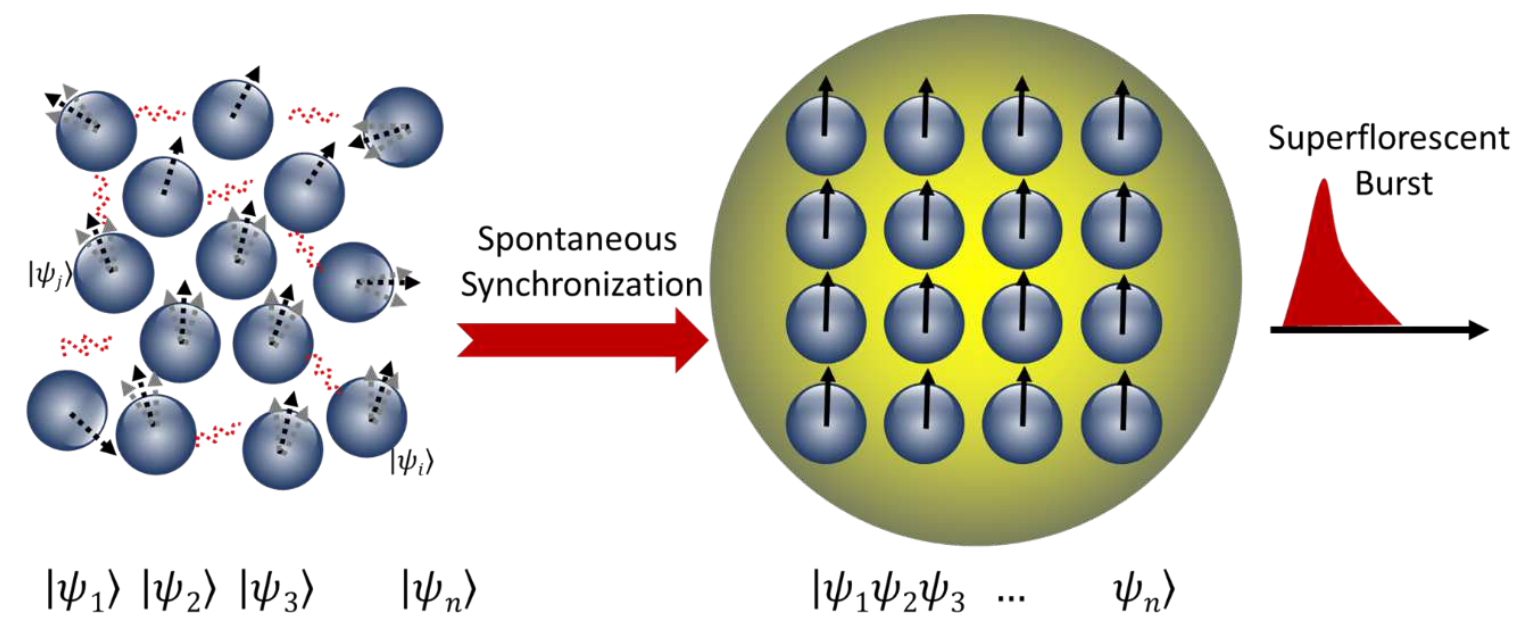

Fig. 1. Graphic representation of superfluorescence evolution. On the left an incoherent ensemble of dipoles is shown. The arrows indicate the randomly distributed phases of individual dipoles. The red waves resemble vacuum fluctuations that leads to spontaneous synchronization. After a time-delay, the phases of the excited dipoles are locked, forming a macroscopic quantum coherent state: "a giant atom" (on the right). This macroscopic state interacts with the radiation field collectively. The system is then described as a single wave function of indistinguishable particles. The collective emission of the macroscopic coherent system leads to a superfluorescent burst. 

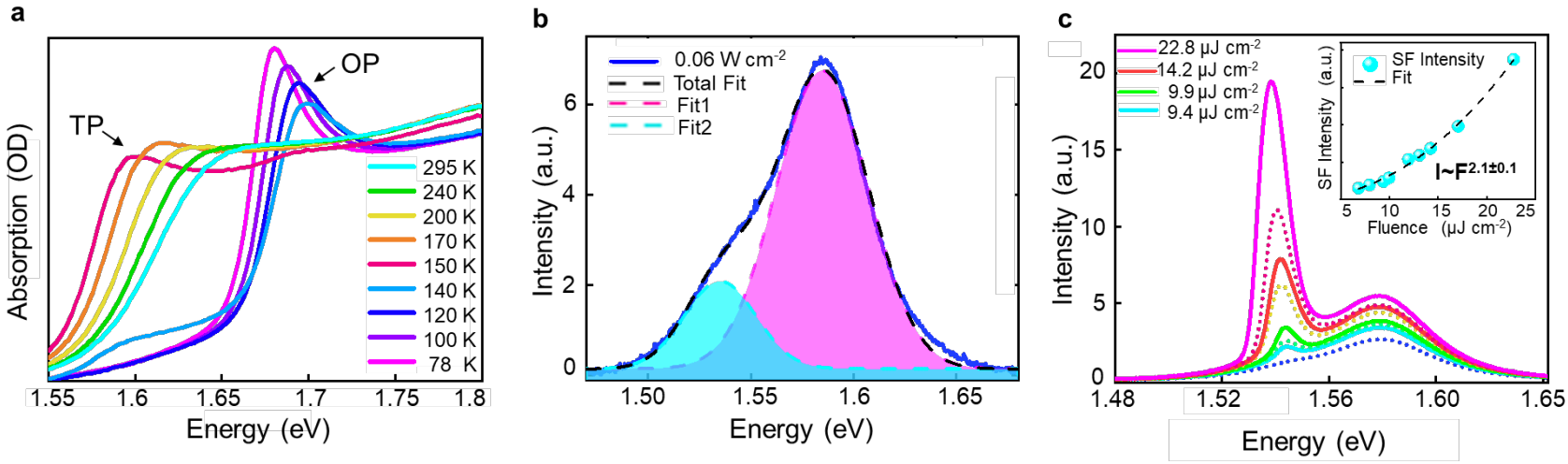

Fig. 2. Optical measurements of $\mathrm{MAPbI}_{3}$ thin films. a, Evolution of the absorption spectra of $\mathrm{MAPbI}_{3}$ from $295 \mathrm{~K}$ to $78 \mathrm{~K}$, indicating a phase transition from TP to OP below $150 \mathrm{~K}$. b, CW PL measured at $78 \mathrm{~K}$. Dashed lines show the two Gaussian fits for spectral features located at $1.54 \mathrm{eV}$ and $1.58 \mathrm{eV}$. c, PL measured at different excitation fluences at $78 \mathrm{~K}$. The dotted lines show the fluences for reference. The inset shows the intensity of the sharp feature extracted using Lorentzian and Gaussian fits for deconvolution. The intensity exhibits a quadratic dependence on excitation fluence with an exponent of $\alpha=2.1 \pm 0.3$. 

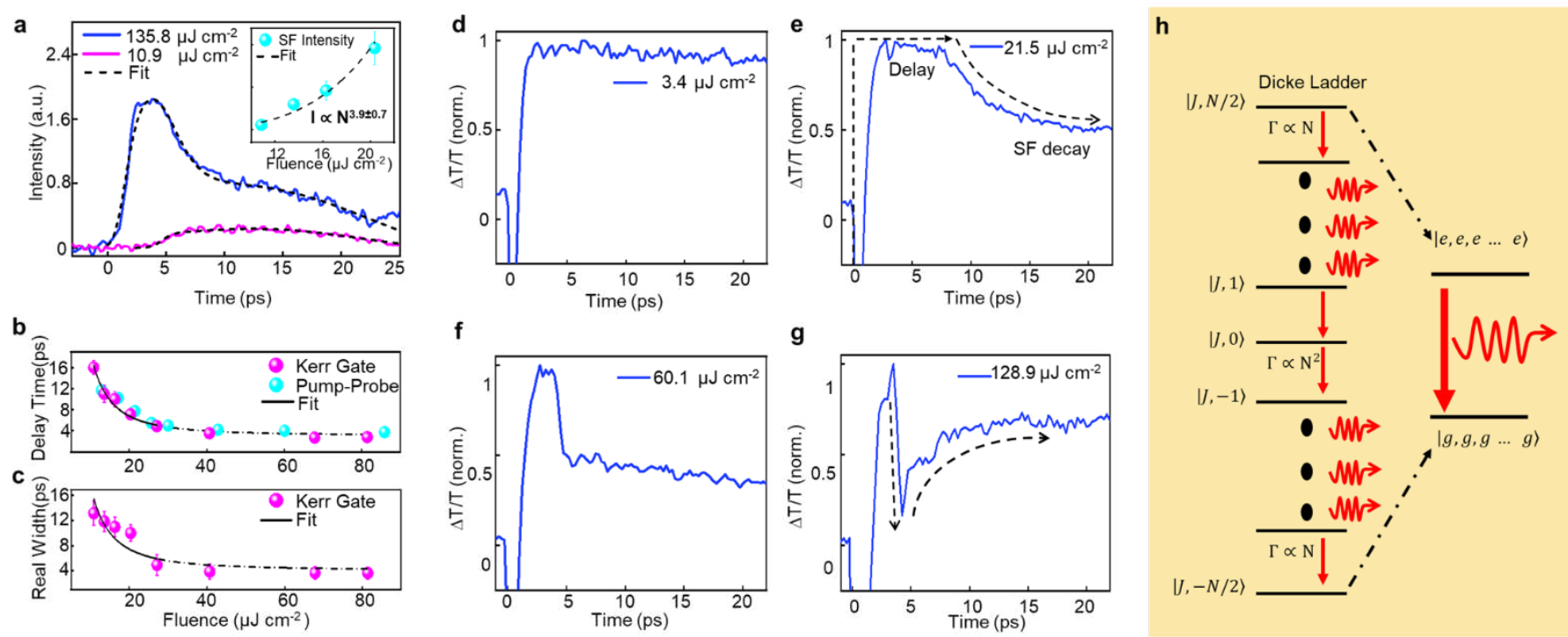

Fig. 3. Time resolved photoluminescence spectra and population dynamics at different excitation fluences. a, Kerr-Gate PL experiment results performed at $78 \mathrm{~K}$. The black dashed lines are the fits to the SF model. The SF peak exhibits a clear time delay $\left(\tau_{D}\right)$ with narrowing peaks. $\mathbf{b}$ and $\mathbf{c}$, The extracted delay time $\left(\tau_{D}\right)$ and the real width $\left(\tau_{R}\right)$ as a function of the excitation fluence, respectively. The black solid lines are the fits according to the theoretical SF model. The cyan circles are the delay time extracted from the pump probe experiment. $\mathbf{d - g}$, show the population dynamics in the TP domains measured using pump-probe experiments probed at $1.54 \mathrm{eV}$ for excitation fluences $3.4 \mu \mathrm{J} \mathrm{cm}^{-2}, 21.5 \mu \mathrm{J} \mathrm{cm}^{-2}, 60.1 \mu \mathrm{J} \mathrm{cm}^{-}$ ${ }^{2}$ and $128.9 \mu \mathrm{J} \mathrm{cm}^{-2}$, respectively. Below the SF threshold (d) the population dynamics show a quick capture of exciton to TP domains and a long monotonic relaxation. Above the SF threshold, the dynamics exhibit distinct intensity-dependent behavior. The population remains steady for a period and then decays at a rate that depends on the fluence. In panel e, the horizontal dashed arrow indicates the delay time before the population starts to relax. This time period shortens with the higher excitation fluences in panels $\mathrm{f}$ and $\mathrm{g}$. The decay of the coherent population is shown by the dashed arrow in e and $\mathrm{g}$. With a slight increase in the excitation fluence the decay rate increases substantially. In panel g the SF decay rate reaches values higher than the exciton capture rate from the OP domains. As a result, SF emission depletes the population in the TP domains for a short time period. $\mathbf{h}$, The coherent population forms a giant atom, with its relaxation path following the states in a Dicke-ladder. 
a

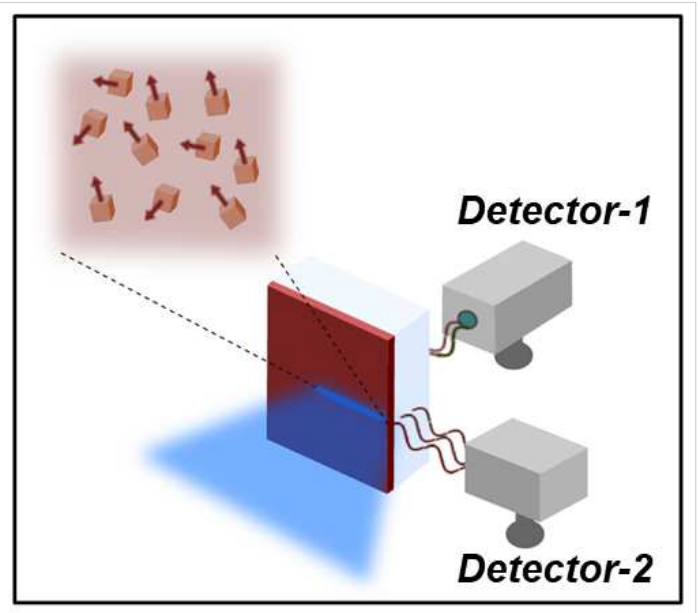

b

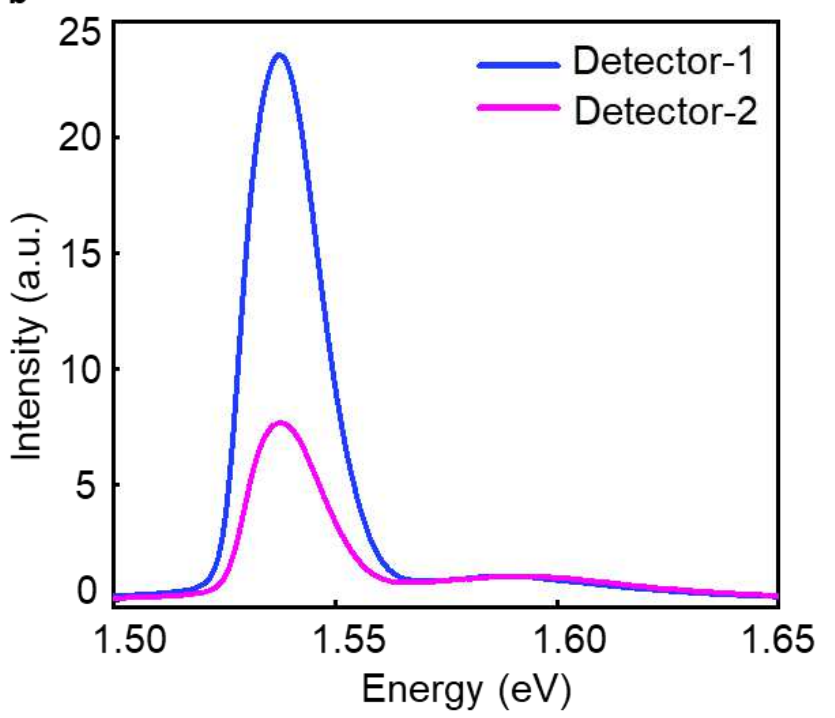

Fig. 4. Direction dependent PL experiment. a, Experimental schematics of direction-dependent PL measurements. The PL emission is collected at two different angles (Detector-1) and (Detector2). b, PL spectra collected at detector positions 1 and 2, at $78 \mathrm{~K}$, normalized with respect to the intensity of the $1.58 \mathrm{eV}$ feature. SF peak intensity is almost 6 times stronger in the forward direction (Detector-1) compared to the edge (Detector-2). The excitation fluence is $17.4 \mu \mathrm{J} \mathrm{cm}^{-2}$. 


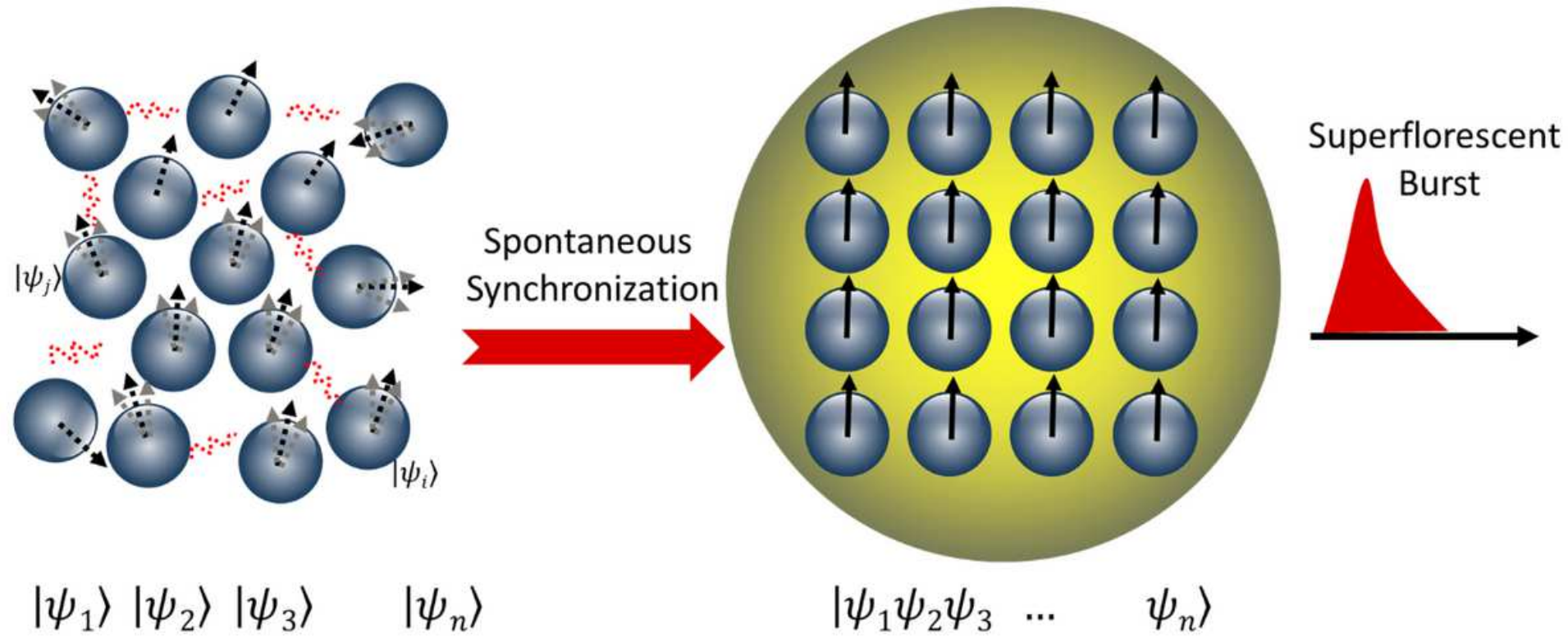

Figure 1

Graphic representation of superfluorescence evolution. On the left an incoherent ensemble of dipoles is shown. The arrows indicate the randomly distributed phases of individual dipoles. The red waves resemble vacuum fluctuations that leads to spontaneous synchronization. After a time-delay, the phases of the excited dipoles are locked, forming a macroscopic quantum coherent state: "a giant atom" (on the right). This macroscopic state interacts with the radiation field collectively. The system is then described as a single wave function of indistinguishable particles. The collective emission of the macroscopic coherent system leads to a superfluorescent burst.
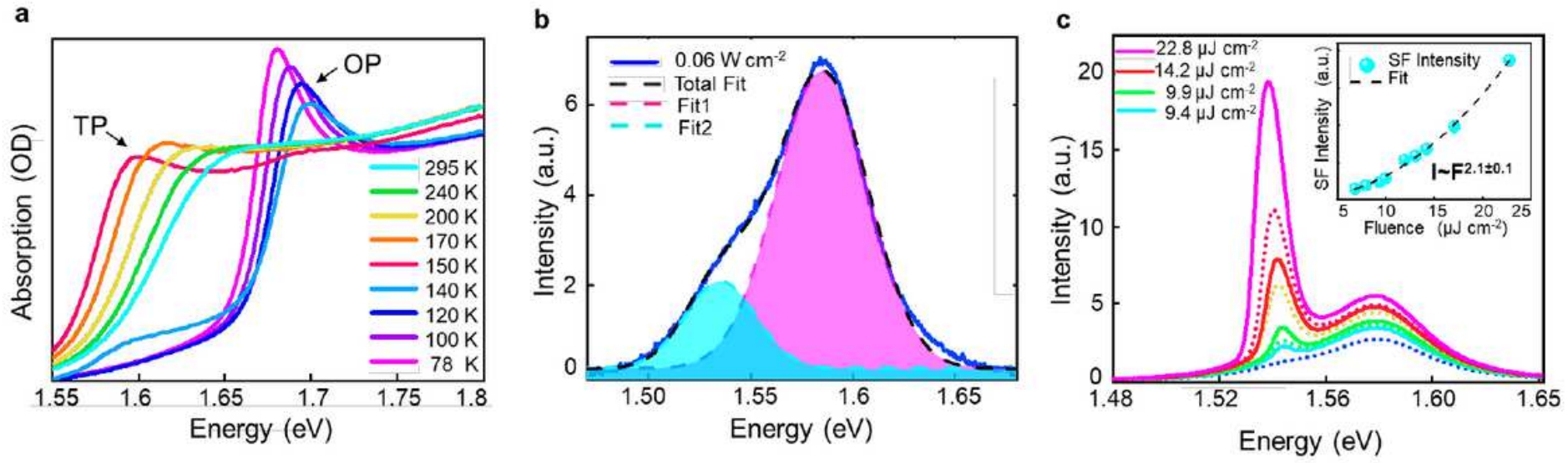

Figure 2

Optical measurements of MAPbl3 thin films. a, Evolution of the absorption spectra of MAPbl3 from $295 \mathrm{~K}$ to $78 \mathrm{~K}$, indicating a phase transition from TP to OP below $150 \mathrm{~K}$. b, CW PL measured at $78 \mathrm{~K}$. Dashed 
lines show the two Gaussian fits for spectral features located at $1.54 \mathrm{eV}$ and $1.58 \mathrm{eV}$. c, PL measured at different excitation fluences at $78 \mathrm{~K}$. The dotted lines show the fluences for reference. The inset shows the intensity of the sharp feature extracted using Lorentzian and Gaussian fits for deconvolution. The intensity exhibits a quadratic dependence on excitation fluence with an exponent of $a=2.1 \pm 0.3$

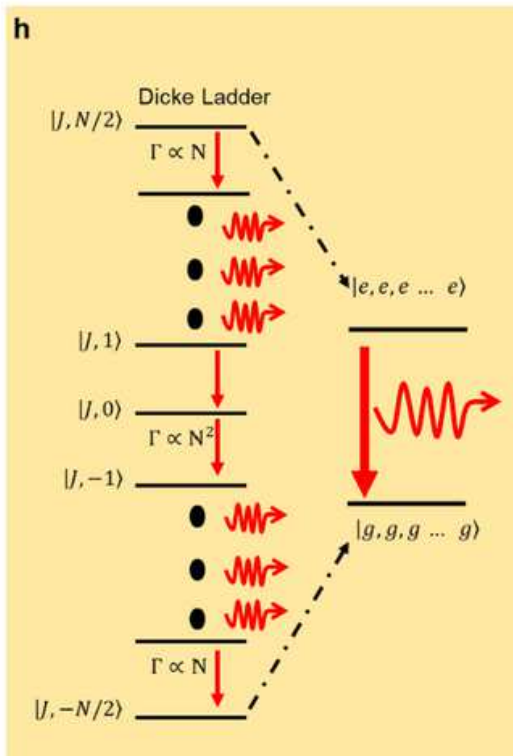

\section{Figure 3}
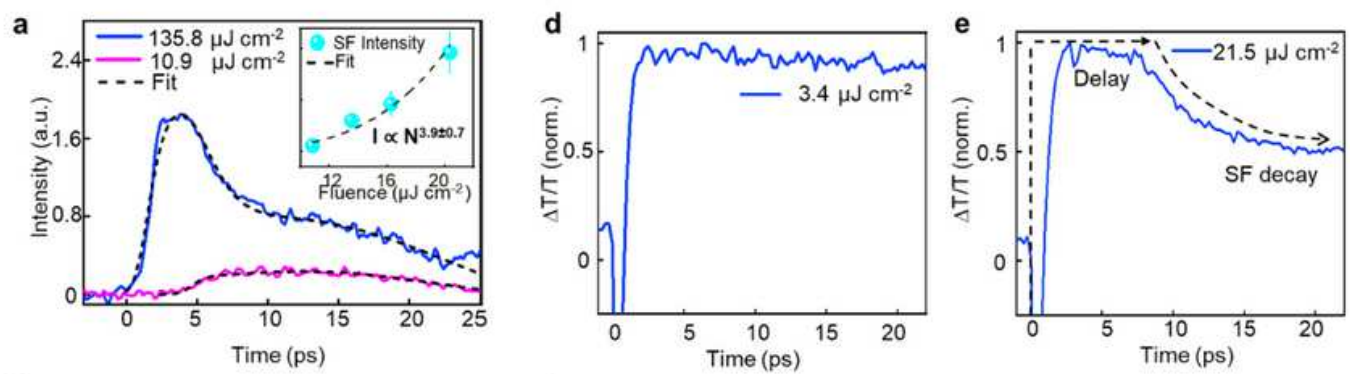

$$
\text { f }
$$

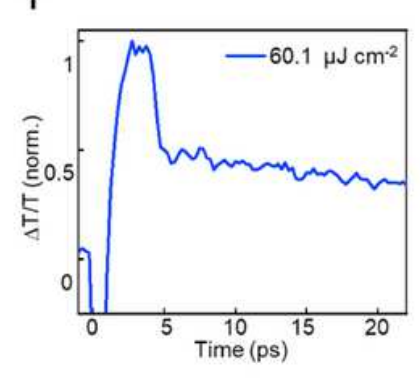

$f$ g

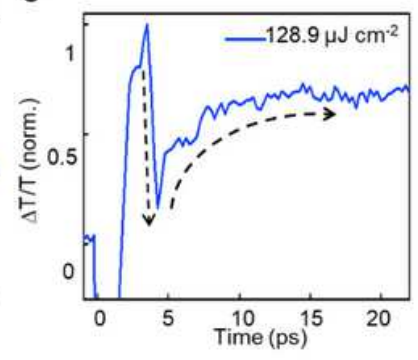

$\mathrm{h}$

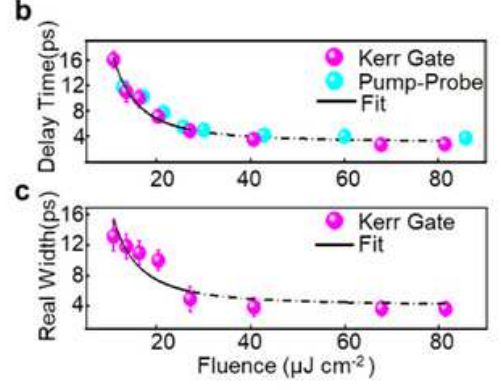

Time resolved photoluminescence spectra and population dynamics at different excitation fluences. a, Kerr-Gate PL experiment results performed at $78 \mathrm{~K}$. The black dashed lines are the fits to the SF model. The SF peak exhibits a clear time delay $(\mathrm{tH})$ with narrowing peaks. $b$ and $c$, The extracted delay time (tH) and the real width ( $\mathrm{t} J$ ) as a function of the excitation fluence, respectively. The black solid lines are the fits according to the theoretical SF model. The cyan circles are the delay time extracted from the pump probe experiment. $d-g$, show the population dynamics in the TP domains measured using pump-probe experiments probed at $1.54 \mathrm{eV}$ for excitation fluences $3.4 \mu \mathrm{J} \mathrm{cm}-2,21.5 \mu \mathrm{J} \mathrm{cm}-2,60.1 \mu \mathrm{J} \mathrm{cm}-2$ and 128.9 $\mu \mathrm{J} \mathrm{cm}-2$, respectively. Below the SF threshold (d) the population dynamics show a quick capture of exciton to TP domains and a long monotonic relaxation. Above the SF threshold, the dynamics exhibit distinct intensity-dependent behavior. The population remains steady for a period and then decays at a rate that depends on the fluence. In panel e, the horizontal dashed arrow indicates the delay time before the population starts to relax. This time period shortens with the higher excitation fluences in panels $f$ and g. The decay of the coherent population is shown by the dashed arrow in e and g. With a slight increase in the excitation fluence the decay rate increases substantially. In panel g the SF decay rate reaches values higher than the exciton capture rate from the OP domains. As a result, SF emission depletes the population in the TP domains for a short time period. $h$, The coherent population forms a giant atom, with its relaxation path following the states in a Dicke-ladder. 
a

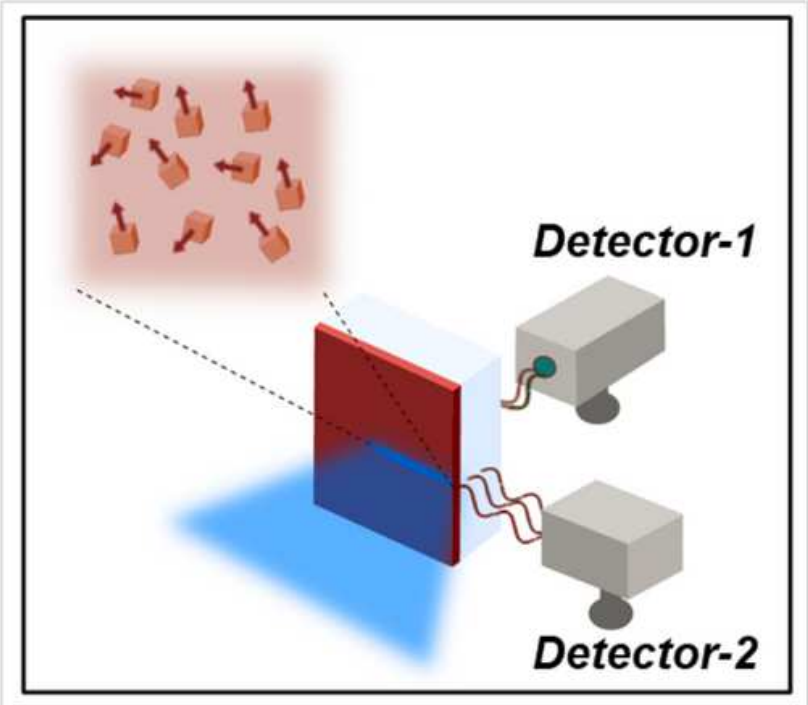

b

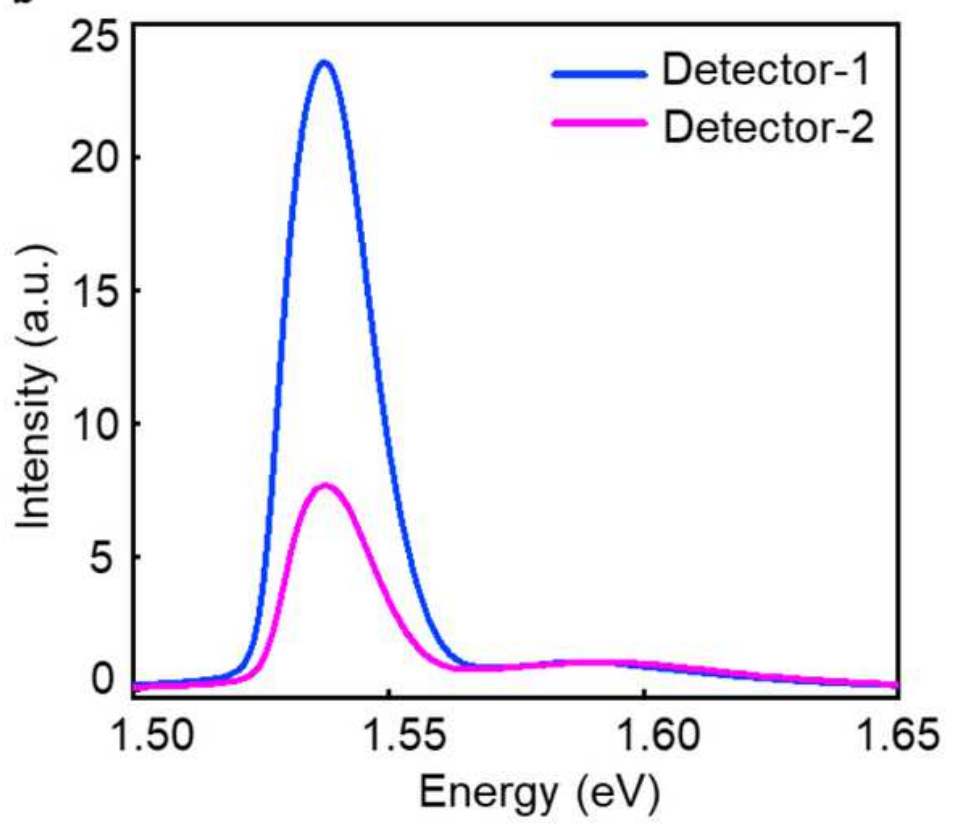

Figure 4

Direction dependent PL experiment. a, Experimental schematics of direction-dependent PL measurements. The PL emission is collected at two different angles (Detector-1) and (Detector-2). b, PL spectra collected at detector positions 1 and 2, at $78 \mathrm{~K}$, normalized with respect to the intensity of the $1.58 \mathrm{eV}$ feature. SF peak intensity is almost 6 times stronger in the forward direction (Detector-1) compared to the edge (Detector-2). The excitation fluence is $17.4 \mu \mathrm{J} \mathrm{cm}-2$.

\section{Supplementary Files}

This is a list of supplementary files associated with this preprint. Click to download.

- SupplementarylnformationSubmission.pdf 